\title{
Improved Confidence Intervals for the Ratio of Coefficients of Variation of Two Lognormal Distributions
}

\author{
Md Sazib Hasan \\ Departmet of Mathematics \\ University of Louisiana at Lafayette \\ Lafayette, LA 70504, USA \\ sazib25@louisiana.edu \\ K. Krishnamoorthy \\ Departmet of Mathematics \\ University of Louisiana at Lafayette \\ Lafayette, LA 70504, USA \\ krishna@louisiana.edu
}

Received 17 January 2016

Accepted 18 April 2017

\begin{abstract}
The problem of estimating the ratio of coefficients of variation of two independent lognormal populations is considered. We propose two closed-form approximate confidence intervals (CIs), one is based on the method of variance estimate recovery (MOVER), and another is based on the fiducial approach. The proposed CIs are compared with another $\mathrm{CI}$ available in the literature. Our new confidence intervals are very satisfactory in terms of coverage properties even for small samples, and better than other CIs for small to moderate samples. The methods are illustrated using an example.
\end{abstract}

Keywords: Coverage probability; Fiducial approach; Index of reliability.

2016 Mathematics Subject Classification: 62F25, 62P10

\section{Introduction}

The coefficient of variation (CV) is defined as the ratio of the standard deviation to the mean, and is a popular measure of variation because it is not affected by the units of measurement. The use of the $\mathrm{CV}$ as an index of reliability or variability in the clinical practice in the context of diagnostic tests, and biochemical laboratory assays has been noted by Orit [13]. This author has also provided examples of use of the $\mathrm{CV}$ as a measure of reliability or variability. For the normal case, the problem of interval estimating the $\mathrm{CV}$ or comparison of two coefficients of variation has been well addressed in the literature. See [1], [15], [5], [4], [3], [14] and [10] and the references therein. The problem of comparing two or more coefficients of variation of two different distributions arises in many practical situations. The $\mathrm{CV}$ is used to judge the precision of measurements or index of reliability, and two measurement methods are compared on the basis of their respective coefficients of variation. 
In financial analysis, the CV has been used as a measure of relative risk, and a test for equality of the coefficients of variation of two stocks is useful to determine whether they involve similar risk; see [11].

In some applications, such as exposure/polution data analysis, measurements are often rightskewed, and lognormal models are commonly postulated for data analysis. To understand the similarity of the problems of estimating the $\mathrm{CV}$ of a lognormal distribution and that of estimating normal parameters, let us consider a random variable $X$ that has a lognormal distribution with parameters $\mu$ and $\sigma^{2}$ so that

$$
\operatorname{mean}(X)=\exp \left(\mu+\sigma^{2} / 2\right) \text { and } \operatorname{var}(X)=\exp \left(\sigma^{2}\right)\left[\exp \left(\sigma^{2}\right)-1\right] \exp (2 \mu) .
$$

The coefficient of variation is given by

$$
\tau=\frac{\sqrt{\operatorname{var}(X)}}{\text { mean }}=\sqrt{\exp \left(\sigma^{2}\right)-1}
$$

We see from the above expression that the problem of estimating the lognormal CV $\tau$ simplifies to the problem of estimating the normal variance based on log-transformed data. Since a logtransformed sample can be regarded as a sample from a normal distribution with mean $\mu$ and variance $\sigma^{2}$, a CI for $\tau$ can be obtained in a straightforward manner. On the other hand, the problem of estimating the ratio of two lognormal coefficients of variation is not a trivial task. Consider two lognormal distributions with parameters $\left(\mu_{1}, \sigma_{1}^{2}\right)$ and $\left(\mu_{2}, \sigma_{2}^{2}\right)$. The ratio of the coefficients of variation of these two distributions is given by

$$
R_{\tau}=\left\{\frac{\exp \left(\sigma_{1}^{2}\right)-1}{\exp \left(\sigma_{2}^{2}\right)-1}\right\}^{\frac{1}{2}}=\frac{\tau_{1}}{\tau_{2}}
$$

where $\tau_{i}=\sqrt{\exp \left(\sigma_{i}^{2}\right)-1}, i=1,2$. It is clear from (3) that testing $R_{r}>1$ simplifies to the problem of testing $\sigma_{1}^{2} / \sigma_{2}^{2}>1$, and an $F$ test based on log-transformed samples can be used to test the variance ratio. However, such test can not be transformed to obtain a confidence interval (CI) for $R_{\tau}$. The problem of finding a CI for the ratio of two lognormal coefficients of variation is addressed in [12]. These authors provided some large sample methods for obtaining CIs for the ratio of coefficients of variation, and an example where two estrogen level measuring methods are compared with respect to coefficient of variation (see Sec. 5).

In this article, we shall propose two simple methods of finding CIs for the ratio of two lognormal coefficients of variation. In the following section, we describe the method of variance estimate recovery (MOVER) in a general setup, and then propose a MOVER CI that is obtained by modifying the CI in [12]. In Sec. 3, we describe the fiducial CI which can be calculated by Monte Carlo simulation or numerically. The accuracy of the CIs and the Nam-Kwon CI are evaluated via Monte Carlo simulation in Sec. 4. Simulation studies indicate that the new CIs are very satisfactory even for small samples. In Sec. 5, the proposed interval estimation methods are illustrated using a real example given in [12]. Some concluding remarks are given in Sec. 6.

\section{MOVER Confidence Intervals}

We shall first describe two approximate CIs based on the MOVER (method of variance estimate discovery) and the fiducial approach. As the MOVER is relatively new, we shall first describe this 
approach as given in [17], [18], and [19]. Consider a set of parameters $\theta_{1}, \ldots ., \theta_{g}$. Let $\widehat{\theta}_{i}$ be an unbiased estimate of $\theta_{i}, i=1, \ldots, g$. Assume that $\widehat{\theta}_{1}, \ldots, \widehat{\theta}_{g}$ are independent. Furthermore, let $\left(l_{i}, u_{i}\right)$ denote the $100(1-\alpha) \%$ confidence interval for $\theta_{i}, i=1, \ldots, k$. The $100(1-\alpha) \%$ MOVER confidence interval $(L, U)$ for $\sum_{i=1}^{k} c_{i} \theta_{i}$ can be expressed as

$$
L=\sum_{i=1}^{g} c_{i} \widehat{\theta}_{i}-\sqrt{\sum_{i=1}^{g} c_{i}^{2}\left(\widehat{\theta}_{i}-l_{i}^{*}\right)^{2}}, \text { with } l_{i}^{*}=\left\{\begin{array}{l}
l_{i} \text { if } c_{i}>0, \\
u_{i} \text { if } c_{i}<0,
\end{array}\right.
$$

and

$$
U=\sum_{i=1}^{g} c_{i} \widehat{\theta}_{i}+\sqrt{\sum_{i=1}^{g} c_{i}^{2}\left(\widehat{\theta}_{i}-u_{i}^{*}\right)^{2}}, \text { with } u_{i}^{*}=\left\{\begin{array}{l}
u_{i} \text { if } c_{i}>0 \\
l_{i} \text { if } c_{i}<0
\end{array}\right.
$$

Graybill and Wang [6] first obtained the above confidence interval for a linear combinations variance components. Zou and co-authors gave a different argument so as to justify the validity of the above confidence interval for any set parameters.

\subsection{MOVER Confidence Intervals for $R_{\tau}$}

Nam-Kwon CI for $R_{\tau}$ Nam and Kwon [12] have proposed several large sample CIs for the ratio of coefficients of variation $R_{\tau}$, and recommended the following MOVER CI that can be obtained from the one for $\ln R_{\tau}=\ln \tau_{1}-\ln \tau_{2}$. To obtain the MOVER CI for $\ln \tau_{1}-\ln \tau_{2}$, these authors have used the asymptotic $\mathrm{CI}$ for $\ln \tau_{i}$ given by

$$
\left(l_{i}, u_{i}\right)=\ln \widehat{\tau}_{i} \pm z_{1-\alpha / 2} \sqrt{\widehat{\operatorname{var}}\left(\ln \widehat{\tau}_{i}\right)}, \quad i=1,2
$$

where $z_{p}$ is the $100 p$ percentile of the standard normal distribution, and

$$
\widehat{\operatorname{var}}\left(\ln \widehat{\tau}_{i}\right)=\frac{\widehat{\sigma}_{i}^{2}\left(1+\widehat{\tau}_{i}^{2}\right)}{2 n_{i} \widehat{\tau}_{i}^{4}}, i=1,2
$$

with $\widehat{\sigma}_{i}^{2}=\left(n_{i}-1\right) S_{i}^{2} / n_{i}$, the maximum likelihood estimate (MLE) of $\sigma_{i}^{2}, i=1,2$. The CIs in (2.3) were obtained using the Wald approach, and they are valid for large samples. In terms of these CIs for $\ln \tau_{i}$, the 100(1- $\left.\alpha\right) \%$ MOVER CI for $\ln \tau_{1}-\ln \tau_{2}$ can be obtained from (2.1) and (2.2) as

$$
L=\ln \widehat{\tau}_{1}-\ln \widehat{\tau}_{2}-\sqrt{\left(\ln \widehat{\tau}_{1}-l_{1}\right)^{2}+\left(\ln \widehat{\tau}_{2}-u_{2}\right)^{2}}
$$

and

$$
U=\ln \widehat{\tau}_{1}-\ln \widehat{\tau}_{2}+\sqrt{\left(\ln \widehat{\tau}_{1}-u_{1}\right)^{2}+\left(\ln \widehat{\tau}_{2}-l_{2}\right)^{2}}
$$

A $100(1-\alpha) \%$ for $R_{\tau}$ on the basis of $(L, U)$ is given by $(\exp (L), \exp (U))$. 


\subsection{An Alternative MOVER CI for $R_{\tau}$}

Notice that Nam and Kwon [12] have used an approximate CIs for $\tau_{i}$ to find the CI in (2.4) and (2.5) for the ratio of coefficients of variation. Instead, we could use the exact CI $\left(l_{i}^{*}, u_{i}^{*}\right)$ for $\tau_{i}^{2}$ given by

$$
l_{i}^{*}=\exp \left[\frac{\left(n_{i}-1\right) S_{i}^{2}}{\chi_{n_{i}-1 ; \alpha}^{2}}\right]-1 \quad \text { and } \quad u_{i}^{*}=\exp \left[\frac{\left(n_{i}-1\right) S_{i}^{2}}{\chi_{n_{i}-1 ; 1-\alpha}^{2}}\right]-1,
$$

where $\chi_{m: p}^{2}$ denotes the $100 p$ percentile of the chi-square distribution with degrees of freedom $m$. Letting $\widehat{\tau}_{i}^{2}=\exp \left(S_{i}^{2}\right)-1$, we can express the MOVER CI $\left(L^{*}, U^{*}\right)$ for $\ln \left(\tau_{1} / \tau_{2}\right)^{2}$ as

$$
L^{*}=\ln \widehat{\tau}_{1}^{2}-\ln \widehat{\tau}_{2}^{2}-\sqrt{\left(\ln \widehat{\tau}_{1}^{2}-\ln l_{1}^{*}\right)^{2}+\left(\ln \widehat{\tau}_{2}^{2}-\ln u_{2}^{*}\right)^{2}}
$$

and

$$
U^{*}=\ln \widehat{\tau}_{1}^{2}-\ln \widehat{\tau}_{2}^{2}+\sqrt{\left(\ln \widehat{\tau}_{1}^{2}-\ln u_{1}^{*}\right)^{2}+\left(\ln \widehat{\tau}_{2}^{2}-\ln l_{2}^{*}\right)^{2}}
$$

In terms of $\left(L^{*}, U^{*}\right)$, the $1-2 \alpha \mathrm{CI}$ for the ratio $\tau_{1} / \tau_{2}$ is given by

$$
\left(\sqrt{\exp \left(L^{*}\right)}, \sqrt{\exp \left(U^{*}\right)}\right)
$$

\section{Fiducial Confidence Intervals}

Consider a sample $Y_{1}, \ldots, Y_{n}$ from a lognormal distribution with parameters $\mu$ and $\sigma^{2}$. Let $X_{i}=\ln \left(Y_{i}\right)$, $i=1, \ldots, n$, so that $X_{i}$ 's are independent $N\left(\mu, \sigma^{2}\right)$ random variables. Define

$$
\bar{X}=\frac{1}{n} \sum_{i=1}^{n} X_{i} \quad \text { and } \quad S^{2}=\frac{1}{n-1} \sum_{i=1}^{n}\left(X_{i}-\bar{X}\right)^{2}
$$

Since the CV of a lognormal distribution is a function of $\sigma^{2}$ only, it is enough to obtain fiducial quantity (FQ) for $\sigma^{2}$ only. Towards this, we first note that $S^{2} \stackrel{d}{=} \sigma^{2} \frac{\chi_{n-1}^{2}}{n-1}$, where $\chi_{m}^{2}$ denotes the chisquare random variable with degrees of freedom (df) $m$ and the notation " $=$ " means "distributed as." Let $s$ be an observed value of $S$. Solving the above equation for $\sigma^{2}$, and replacing $S$ with $s$, we obtain the fiducial quantity for $\sigma^{2}$ as

$$
Q_{\sigma^{2}}=\frac{(n-1) s^{2}}{\chi_{n-1}^{2}}
$$

Notice that, for a fixed $s^{2}$, the fiducial distribution of $\sigma^{2}$ does not depend on any unknown parameters. A fiducial quantity for a real-valued function, say, $f\left(\sigma^{2}\right)$ can be obtained by simple substitution as $f\left(Q_{\sigma^{2}}\right)$. For the present problem, let $s_{i}^{2}$ be an observed value of $S_{i}^{2}, i=1,2$. Then a FQ for $\tau_{i}^{2}$ 
can be expressed as

$$
Q_{\tau_{i}^{2}}=\exp \left(Q_{\sigma}^{2}\right)-1=\exp \left(\frac{\left(n_{i}-1\right) s_{i}^{2}}{\chi_{n_{i}-1}^{2}}\right)-1, i=1,2 .
$$

A FQ for $R_{\tau}^{2}=\tau_{1}^{2} / \tau_{2}^{2}$ can be obtained by substitution, and is given by

$$
Q_{R_{\tau}^{2}}=\frac{\exp \left(Q_{\tau_{1}^{2}}\right)-1}{\exp \left(Q_{\tau_{2}^{2}}\right)-1}=\frac{\exp \left[\left(n_{1}-1\right) s_{1}^{2} / \chi_{n_{1}-1}^{2}\right]-1}{\exp \left[\left(n_{2}-1\right) s_{2}^{2} / \chi_{n_{2}-1}^{2}\right]-1} .
$$

For a given $\left(s_{1}^{2}, s_{2}^{2}\right)$, the distribution of $Q_{R_{\tau}^{2}}$ does not depend on any parameters, and so the percentiles of $Q_{R_{\tau}^{2}}$ can be estimated by Monte Carlo simulation. The lower $\alpha$ quantile and the upper $\alpha$ quantile of $Q_{R_{\tau}^{2}}$ form a $1-2 \alpha \mathrm{CI}$ for $R_{\tau}^{2}$, from which a CI for $R_{\tau}$ can be readily obtained.

To avoid using simulation to estimate the percentiles of $Q_{R_{\tau}^{2}}$, we could use the modified normalbased approximation given in [9]. To apply this approximation, let

$$
T_{i}=\exp \left[\frac{\left(n_{i}-1\right) s_{i}^{2}}{\chi_{n_{i}-1}^{2}}\right]-1, \text { and } T_{i ; \alpha}=\exp \left[\frac{\left(n_{i}-1\right) s_{i}^{2}}{\chi_{n_{i}-1 ; 1-\alpha}^{2}}\right]-1, \quad i=1,2,
$$

where $0<\alpha<1$. The lower $\alpha$ quantile $L^{\prime}$ and the upper $\alpha$ quantile $U^{\prime}$ of $\ln Q_{R_{\tau}^{2}}$ can be approximated as

$$
L^{\prime}=\ln T_{1 ; .5}-\ln T_{2 ; .5}-\sqrt{\left(\ln T_{1 ; .5}-\ln T_{1 ; \alpha}\right)+\left(\ln T_{2 ; .5}-\ln T_{2 ; 1-\alpha}\right)^{2}}
$$

and

$$
U^{\prime}=\ln T_{1 ; .5}-\ln T_{2 ; .5}+\sqrt{\left(\ln T_{1 ; .5}-\ln T_{1 ; 1-\alpha}\right)^{2}+\left(\ln T_{2 ; .5}-\ln T_{2 ; \alpha}\right)^{2}} .
$$

It should be noted that approximation proposed by Krishnamoorthy [9] involves expectations of the random variables rather than medians as given in $L^{\prime}$ and $U^{\prime}$. For our present set up, evaluation of $E\left(T_{i}\right)$ is essentially the same as that for the moment generating function of an inverted chi-square random variable, which does not exist. So $E\left(\ln \left(T_{i}\right)\right)$ does not exist. Instead of expectations, we use the median of $\ln T_{i}$, and later verify its validity in terms of coverage probabilities of CIs based on $\left(L^{\prime}, U^{\prime}\right)$. After finding $\left(L^{\prime}, U^{\prime}\right)$, we can express the $1-2 \alpha \mathrm{CI}$ for the ratio $\tau_{1} / \tau_{2}$ as

$$
\left(\sqrt{\exp \left(L^{\prime}\right)}, \sqrt{\exp \left(U^{\prime}\right)}\right)
$$

and we shall refer to this CI as approximate fiducial CI.

\section{Simulation Studies}

To judge the performance of the proposed CIs in the preceding sections, we estimated the coverage probabilities for some assumed values of parameters $\left(\sigma_{1}, \sigma_{2}\right)$ and sample sizes varying from small to moderate. In practical applications, the values of $\left(\sigma_{1}, \sigma_{2}\right)$, which are standard deviations of log-transformed variables, are usually small, and so we consider small values of $\left(\sigma_{1}, \sigma_{2}\right)$ in our simulation studies. For each interval estimation method, the coverage probabilities were estimated using simulation consisting of 100,000 runs. 
Table 1. Coverage probabilities and tail error rates of $95 \%$ CIs as function of parameters

\begin{tabular}{cc|cccccccccccc}
\multicolumn{1}{c}{$E R_{L}=$ error rates on left; $\mathrm{CP}=$ coverage probability; $E R_{R}=$ error rates on right } \\
\hline \multirow{3}{*}{$\left(n_{1}, n_{2}\right)$} & $\mathrm{CI}$ & \multicolumn{2}{c}{$\left(\sigma_{1}, \sigma_{2}\right)=(.1, .3)$} & \multicolumn{3}{c}{$\left(\sigma_{1}, \sigma_{2}\right)=(.3, .7)$} & \multicolumn{3}{c}{$\left(\sigma_{1}, \sigma_{2}\right)=(.4,1.2)$} & \multicolumn{3}{c}{$\left(\sigma_{1}, \sigma_{2}\right)=(.5,1.6)$} \\
\hline$(4,8)$ & 1 & 2.2 & 86.5 & 11.3 & 2.5 & 87.7 & 9.8 & 3.9 & 90.5 & 5.6 & 6.2 & 91.9 & 1.9 \\
& 2 & 2.2 & 95.5 & 2.3 & 2.0 & 95.6 & 2.4 & 1.8 & 95.5 & 2.7 & 1.8 & 95.4 & 2.8 \\
& 3 & 2.5 & 95.0 & 2.5 & 2.3 & 95.1 & 2.6 & 2.1 & 95.0 & 2.9 & 2.1 & 94.9 & 3.0 \\
$(5,10)$ & 1 & 2.1 & 88.5 & 9.4 & 2.4 & 89.6 & 8.0 & 3.8 & 91.7 & 4.4 & 5.9 & 92.7 & 1.4 \\
& 2 & 2.3 & 95.4 & 2.3 & 2.1 & 95.5 & 2.4 & 2.0 & 95.2 & 2.8 & 2.9 & 95.2 & 2.8 \\
& 3 & 2.5 & 95.0 & 2.5 & 2.3 & 95.1 & 2.6 & 2.2 & 94.8 & 2.9 & 2.2 & 94.9 & 2.9 \\
$(10,5)$ & 1 & 10.1 & 88.2 & 1.7 & 11.2 & 88.0 & 0.8 & 14.7 & 85.3 & 0.0 & 18.9 & 81.0 & 0.0 \\
& 2 & 2.3 & 95.3 & 2.4 & 2.3 & 95.0 & 2.7 & 2.2 & 94.7 & 3.1 & 2.3 & 94.6 & 3.1 \\
& 3 & 2.4 & 94.9 & 2.7 & 2.4 & 94.7 & 2.9 & 2.3 & 94.8 & 2.9 & 2.4 & 94.6 & 3.0 \\
$(7,12)$ & 1 & 2.3 & 91.0 & 6.7 & 2.7 & 91.7 & 5.5 & 4.3 & 93.2 & 2.5 & 6.5 & 92.8 & 0.1 \\
& 2 & 2.2 & 95.5 & 2.3 & 2.2 & 95.4 & 2.4 & 2.0 & 95.3 & 2.7 & 2.1 & 94.8 & 3.0 \\
& 3 & 2.4 & 95.1 & 2.5 & 2.4 & 95.1 & 2.5 & 2.2 & 94.9 & 2.8 & 2.3 & 94.8 & 2.5 \\
$(10,10)$ & 1 & 4.0 & 92.2 & 3.7 & 4.7 & 92.6 & 2.7 & 7.0 & 92.4 & 0.6 & 10.0 & 89.9 & 0.1 \\
& 2 & 2.2 & 95.4 & 2.4 & 2.2 & 95.3 & 2.6 & 2.2 & 94.8 & 3.1 & 2.2 & 94.8 & 3.0 \\
& 3 & 2.5 & 95.0 & 2.5 & 2.3 & 95.0 & 2.7 & 2.2 & 94.6 & 3.1 & 2.4 & 94.7 & 2.9 \\
$(10,15)$ & 1 & 2.5 & 92.5 & 4.9 & 2.9 & 92.9 & 4.2 & 4.6 & 93.6 & 1.7 & 6.6 & 93.0 & 0.4 \\
& 2 & 2.3 & 95.4 & 2.3 & 2.2 & 95.3 & 2.6 & 2.2 & 95.0 & 2.8 & 2.1 & 94.9 & 3.0 \\
& 3 & 2.4 & 95.1 & 2.5 & 2.3 & 95.0 & 2.7 & 2.3 & 94.8 & 2.9 & 2.2 & 94.9 & 2.7 \\
& 1 & 3.2 & 93.6 & 3.2 & 3.7 & 94.0 & 2.3 & 5.2 & 93.8 & 1.0 & 7.2 & 92.5 & 0.3 \\
& 2 & 2.3 & 95.2 & 2.5 & 2.4 & 95.0 & 2.6 & 2.1 & 95.0 & 2.9 & 2.3 & 94.8 & 2.9 \\
& 3 & 2.5 & 95.0 & 2.5 & 2.4 & 95.0 & 2.6 & 2.3 & 94.8 & 2.9 & 2.3 & 94.9 & 2.9 \\
\hline
\end{tabular}

${ }^{1} \mathrm{Nam}$ and Kwon CI in (2.4) and (2.5)

${ }^{2}$ The modified MOVER CI in (2.8)

${ }^{3}$ Approximate fiducial $\mathrm{CI}$ in (3.7)

The estimated coverage probabilities of $95 \% \mathrm{CI}$ by the Nam-Kwon method given in (2.4) and (2.5), the enhanced MOVER CI in (2.8) and the approximate fiducial CI (3.7) are reported in Tables 1 and 2. We also tabulated tail-error rates for all three CIs along with coverage probabilities. Examination of coverage probabilities and tail-error rates clearly indicate that the large sample CIs by Nam and Kwon [12] are accurate only for very large samples. For large samples, the coverage probabilities of Nam-Kwon CIs are close to the nominal level, but the tail-error rates are not balanced, and error rate in one tail is larger than the one in the other tail. For example, when $\left(n_{1}, n_{2}\right)=(20,20)$, the error rates on the left tail are larger than those in the right tail when $\sigma_{1}$ is considerably smaller than $\sigma_{2}$. See also error rates for $\left(n_{1}, n_{2}\right)=(30,40)$ in Table 2 . On the other hand, the enhanced MOVER CI (2.8) and the approximate fiducial CI (3.7) control the coverage probabilities very close to the nominal level .95 for all sample sizes and parameter configurations considered. Even for sample sizes $(5,5)$ (see Table 2), the error rates on tails are not appreciably different and the coverage probabilities are very close to the nominal level. Between the MOVER CI (2.8) and the fiducial CI (3.7), the latter one controls the coverage probabilities and error rates on both tails better than the former. 
Table 2. Coverage probabilities and tail error rates of $95 \%$ CIs as function of sample sizes

\begin{tabular}{cc|cccccccccccc}
\multicolumn{10}{c}{$E R_{L}=$ error rates on left; $\mathrm{CP}=$ coverage probability; $E R_{R}=$ error rates on right } \\
\hline \multirow{3}{*}{$\left(\sigma_{1}, \sigma_{2}\right)$} & $\mathrm{CI}$ & $\left.E R_{L}, n_{2}\right)=(5,5)$ & $\mathrm{CP}$ & $E R_{R}$ & $E R_{L}$ & $\mathrm{CP}$ & $E R_{R}$ & $E R_{L}$ & $\mathrm{CP}$ & $E R_{R}$ & $E R_{L}$ & $\mathrm{CP}$ & $E R_{R}$ \\
\hline$(.1, .7)$ & 1 & 7.3 & 88.8 & 3.8 & 5.0 & 92.5 & 2.5 & 2.4 & 92.7 & 4.8 & 2.9 & 94.4 & 2.7 \\
& 2 & 2.0 & 95.4 & 2.6 & 2.2 & 95.3 & 2.5 & 2.2 & 95.3 & 2.5 & 2.3 & 95.1 & 2.6 \\
& 3 & 2.2 & 95.1 & 2.7 & 2.3 & 95.0 & 2.7 & 2.3 & 95.1 & 2.6 & 2.3 & 95.0 & 2.6 \\
$(.3, .9)$ & 1 & 8.0 & 89.4 & 2.6 & 5.5 & 92.7 & 1.8 & 2.6 & 93.1 & 4.3 & 3.1 & 94.5 & 2.4 \\
& 2 & 2.0 & 95.4 & 2.6 & 2.1 & 95.2 & 2.7 & 2.1 & 95.3 & 2.6 & 2.2 & 95.1 & 2.7 \\
& 3 & 2.3 & 94.8 & 2.9 & 2.2 & 95.0 & 2.8 & 2.3 & 95.0 & 2.7 & 2.3 & 95.0 & 2.7 \\
$(.5,1.4)$ & 1 & 11.5 & 88.0 & 0.5 & 8.0 & 91.8 & 0.2 & 3.8 & 94.4 & 2.8 & 4.4 & 94.5 & 1.1 \\
& 2 & 2.0 & 95.0 & 3.0 & 2.0 & 95.0 & 3.0 & 2.1 & 95.0 & 2.9 & 2.3 & 94.9 & 2.8 \\
& 3 & 2.2 & 94.6 & 3.2 & 2.3 & 94.7 & 3.0 & 2.1 & 94.9 & 2.8 & 2.2 & 95.0 & 2.8 \\
$(.3,1.5)$ & 1 & 13.3 & 86.4 & 0.1 & 9.5 & 90.4 & 0.1 & 4.9 & 94.0 & 1.1 & 5.0 & 94.1 & 0.9 \\
& 2 & 2.0 & 94.6 & 3.4 & 2.1 & 94.7 & 3.1 & 2.1 & 94.8 & 1.1 & 2.1 & 94.9 & 2.8 \\
& 3 & 2.2 & 94.2 & 3.6 & 2.1 & 94.7 & 3.1 & 2.3 & 94.7 & 3.0 & 2.3 & 94.9 & 2.8 \\
$(.9,1.8)$ & 1 & 13.6 & 86.3 & 0.0 & 9.7 & 90.3 & 0.0 & 4.5 & 94.7 & 0.8 & 5.0 & 94.4 & 0.6 \\
& 2 & 2.1 & 95.3 & 2.6 & 2.1 & 95.1 & 2.8 & 2.1 & 95.2 & 2.7 & 2.3 & 94.9 & 2.6 \\
& 3 & 2.3 & 95.0 & 2.7 & 2.3 & 95.0 & 2.7 & 2.5 & 95.0 & 2.5 & 2.4 & 95.0 & 2.6 \\
& 1 & 5.8 & 89.0 & 5.2 & 4.0 & 92.5 & 3.5 & 1.7 & 92.4 & 5.9 & 2.5 & 94.2 & 3.2 \\
& 2 & 2.1 & 95.7 & 2.2 & 2.3 & 95.4 & 2.3 & 2.3 & 95.2 & 2.4 & 2.4 & 95.1 & 2.5 \\
& 3 & 2.4 & 95.1 & 2.5 & 2.4 & 95.1 & 2.5 & 2.5 & 95.0 & 2.5 & 2.5 & 95.0 & 2.5 \\
\hline
\end{tabular}

\footnotetext{
${ }^{1} \mathrm{Nam}$ and Kwon CI in (2.4) and (2.5)

${ }^{2}$ The modified MOVER CI in (2.8)

${ }^{3}$ Approximate fiducial CI in (3.7)
}

Overall, on the basis of simplicity and coverage probabilities, the approximate fiducial CI (3.7) is preferable to other two CIs for applications.

\section{An Example}

To illustrate interval estimation methods described in the preceding sections, we shall use the example given in [12]. The radioimmuno assay (RIA) method has been routinely used for its efficiency and low cost in measuring estrogen metabolites in blood and urine. Faupel-Badger et al. [2] have compared concentrations of estrogen metabolites by RIA with the concentrations obtained using a novel and high-performance liquid chromatography-tandem mass spectrometry (LC-MS/MS). For the purpose of illustration, Nam and Kwon have used the quality control data of 16 measurements by RIA method from each of 3 postmenopausal women, and 28 measurements from a postmenopausal woman using LC-MS/MS, and 25 measurements obtained from another postmenopausal woman using LC-MS/MS. The estrogen measurements by RIA were obtained in (micrograms/liter), and those by LC-MS/MS were obtained in picagram per 0.05 milliliter. These two methods are compared with respect to the coefficient variation, which is an index of reliability of an the assay method. The method with a smaller CV is more reliable than the one with a larger CV. It is commonly 
assumed that estrone measurements follow log normal models, and so all measurements were logtransformed. The calculated statistics for these two sets of log-transformed measurements are as follows.

\begin{tabular}{llll}
\hline RIA: & $n_{1}=48$, & $s_{1}^{2}=0.029065$, & $\widehat{\tau}_{1}=0.17173$ \\
LC-MS/MS: & $n_{2}=53$, & $s_{2}^{2}=0.0032192$, & $\widehat{\tau}_{2}=0.05678$ \\
\hline
\end{tabular}

It should be noted that the reported point estimates $\widehat{\tau}_{1}^{*}=.1699$ and $\widehat{\tau}_{2}^{*}=.0562$ in Nam and Kwon's paper are based on the MLEs $\widehat{\sigma}_{1}=.1687$ and $\widehat{\sigma}_{2}=.0562$.

Nam and Kwon's (2016) 95\% MOVER CI formed by (2.4) and (2.5) for the ratio of coefficients of variation is $(2.29,3.99)$. To find the new MOVER CI on the basis of (2.6) and (2.7), we found

$$
\chi_{47 ; .025}^{2}=29.9562, \chi_{47 ;, 975}^{2}=67.8207, \chi_{52 ; .025}^{2}=33.9681 \text {, and } \chi_{52 ; .975}^{2}=73.8099 .
$$

Using these percentiles in (2.6) and (2.7), we calculated the MOVER CI in $(2.8)$ as $(2.28,4.04)$. The approximate fiducial CI (3.7) is computed similarly, and is $(2.28,4.03)$. As the sample sizes are considerably large, all three methods produced similar CIs. All these CIs indicate that the CV for RIA is about two to four times larger than the CV for LC-MS/MS, and so the LC-MS/MS method is highly reliable compared to the RIA.

\section{Concluding Remarks}

In this article, we have provided two simple CIs for the ratio of coefficients of variation from lognormal distributions. One CI is based on the MOVER, which is a new general method, and the other $\mathrm{CI}$ is based on the fiducial approach. Even though the fiducial approach was introduced in 1930s, it was not a popular approach until publication of the papers [16], [7] and [8]. These authors have promoted the fiducial approach by noting fiducial solutions to problems where the standard approach fail to produce accurate results. We have provided fiducial solution to yet another problem of practical importance. Finally, we note that the fiducial CI is very simple, and they can be calculated using a scientific calculator or $\mathrm{R}$ in a straightforward manner.

\section{Acknowledgments}

The authors are grateful to the editor and a reviewer for providing comments and suggestions

\section{References}

[1] N.L. Johnson and B.L. Welch, Application of the nonecentral t-distribution, Biometrika 31 (1940) 362389.

[2] J.M. Faupel-Badger, B.J. Fuhrman, X. Xu, R.T. Falk, L.K. Keefer, T.D. Veenstra, R.N. Hoover and R.G. Ziegler, Comparison of liquid chromatographytandem mass spectrometry, RIA, and ELISA methods for measurement of urinary estrogens. Cancer Epidemiology Biomarkers \& Prevention, 19 (2010) 292300.

[3] G.J. Feltz and G.E. Miller, An asymptotic test for the equality of coefficients of variation from $k$ normal populations, Statistics in Medicine, 15 (1996) 647-658.

[4] J. Forkman, Estimator and tests for common coefficients of variation in normal distributions, Соттиnications in Statistics - Theory and Methods 38 (2009) 233-251.

[5] Fung, W.K. and Tsang, T.S. (1998). A simulation study comparing tests for the equality of coefficients of variation. Statistics in Medicine, 17, 2003-2014.

[6] F.A. Graybill, and C-M. Wang, Confidence intervals on nonnegative linear combinations of variances, Journal of the American Statistical Association 75 (1980) 869-873. 
[7] J. Hannig H.K. Iyer, and P. Patterson, Fiducial generalized confidence intervals. Journal of the American Statistical Association 101 (2006) 254-269.

[8] J. Hannig, On generalized fiducial inference, Statistica Sinica 19 (2009) 491-544.

[9] K. Krishnamoorthy, Modified normal-based approximation to the percentiles of linear combination of independent random variables with applications. Communications in Statistics - Simulation and Computation 45 (2016) 2428-2444.

[10] K. Krishnamoorthy and M. Lee, Improved tests for the equality of normal coefficients of variation, Computational Statistics 29 (2013) 215-232.

[11] E.G. Miller and M.J. Karson, Testing the equality of two coefficients of variation, American Statistical Association : Proceedings of the Business and Economics Section, Part I, (1977) 278-283.

[12] J-M. Nam and D. Kwon, Inference on the ratio of two coefficients of variation of two lognormal distributions, Communications in Statistics - Theory and Methods 46 (2017) 8575-8587.

[13] S. Orit, The Coefficient of Variation as an Index of Measurement Reliability. Methods of Clinical Epidemiology S.A.R. Doi and G.M. Williams (eds.)(Springer-Verlag, Berlin, 2013)

[14] L. Tian, Inferences on the common coefficient of variation. Statistics in Medicine 24 (2005) 2213-2220.

[15] M.G. Vangel, Confidence intervals for a normal coefficient of variation. The American Statistician $\mathbf{5 0}$ (1996) 21-26.

[16] S. Weerahandi, Generalized confidence intervals. Journal of the American Statistical Association 88 (1993) 899-905.

[17] G.Y. Zou and A. Donner, Construction of confidence limits about effect measures: A general approach, Statistics in Medicine 27 (2008) 1693-1702.

[18] G.Y. Zou, C.Y. Huo and J. Taleban, Confidence interval estimation for lognormal data with application to health economics. Computational Statistics and Data Analysis 53 (2009) 3755-3764.

[19] G.Y. Zou, J. Taleban and C.Y. Huo, Simple confidence intervals for lognormal means and their differences with environmental applications. Environmetrics 20 (2009) 172-180. 